Article

\title{
The use of clinical prediction rules without echocardiographic evaluation in Staphylococcus aureus bacteraemia may not be enough to exclude infective endocarditis and be associated with increased mortality
}

Jorge Calderón-Parra 1,2,*, Itziar Diego-Yagüe 1, Beatriz Santamarina-Alcantud 3, Alberto Mora-Vargas ${ }^{1}$, José Manuel Vázquez-Comendador ${ }^{1}$, Ana Fernandez-Cruz ${ }^{1,2}$, Elena Muñez-Rubio ${ }^{1,2}$, Andrea Gutiérrez-Villanueva ${ }^{1}$, Isabel Sánchez-Romero ${ }^{3}$ and Antonio Ramos-Martínez ${ }^{1,2}$

1 Infectious Diseases Unit, Service of Internal Medicine, Hospital Universitario Puerta de Hierro, Majadahonda, Majadahonda, Spain.

2 Investigational institute Puerta de Hierro hospital Segovia de Aranda (IDIPDHSA)

3 Microbiology Service, Hospital Universitario Puerta de Hierro, Majadahonda, Majadahonda, Spain.

* Correspondence: jorge050390@gmail.com;

\begin{abstract}
Background. It is unclear whether the use of clinical prediction rules is sufficient to rule out infective endocarditis (IE) in patients with Staphylococcus aureus bacteremia (SAB) without an echocardiogram evaluation, either transthoracic (TTE) and/or transesophageal (TEE). Our primary purpose was to test the usefulness of PREDICT, POSITIVE and VIRSTA scores to rule out IE without echocardiography. Our secondary purpose was to evaluate whether not performing an echocardiogram evaluation is associated with higher mortality. Methods. We conducted a unicentric retrospective cohort including all patients with a first SAB episode from January 2015 to December 2020. IE was defined according to modified Duke criteria. We predefined threshold cut-off points to consider that IE was ruled out by means of the mentioned scores. To assess 30-day mortality, we used a multivariable regression model considering performing an echocardiogram as covariate. Results. Out of 404 patients, IE was diagnosed in 50 (12.4\%). Prevalence of IE within patients with negative PREDICT, POSITIVE and VIRSTA scores was: 3.6\% (95\% CI 0.1-6.9\%), 4.9\% (95\% CI 2.2-7.7\%), and $2.2 \%$ (95\% CI $0.2-4.3 \%$ ), respectively. Patients with negative VIRSTA and negative TTE had an IE prevalence of $0.9 \%$ (95\% CI 0-2.8\%). Performing an echocardiogram was independently associated with lower 30-day mortality (OR 0.24 95\%CI 0.10-0.54, $p=0.001$ ). Conclusion. PREDICT and POSITIVE scores were not sufficient to rule out IE without TEE. In patients with negative VIRSTA score, it was doubtful if IE could be discarded with a negative TTE. Not performing an echocardiogram was associated with worse outcomes, which might be related to presence of occult IE. Further studies are needed to assess the usefulness of clinical prediction rules in avoiding echocardiographic evaluation in SAB patients.
\end{abstract}

Keywords: staphylococcus aureus; endocarditis; clinical prediction rules; echocardiography

\section{Introduction}

Staphylococcus aureus (SA) bacteremia (SAB) is one of the most frequent causes of positive blood cultures $(\mathrm{BC})$ and has a high mortality $(1,2)$. One of the most serious complications in patients with $\mathrm{SAB}$ is the development of infective endocarditis (IE), which worsens the prognosis $(2,3)$. IE in patients with $\mathrm{SAB}$ can occur in the absence of risk factors and with structurally normal heart valves (4).

The diagnosis of IE in patients with SAB is of vital importance because it modifies the antibiotic selection and duration (5) and cardiac surgery may be necessary $(6,7)$. Some 
authors have estimated that not reaching a diagnosis of IE in patients with SAB conveys a $15-20 \%$ increase in mortality (8). Therefore, systematic echocardiography has been classically recommended, with transesophageal echocardiography (TEE) being preferred to transthoracic echocardiography (TTE). This recommendation appears in the current guidelines (9) with a grade of evidence A-II.

However, this increased mortality should be weighed against the risks induced by routine TEE. Several studies have shown that serious complications of the performance of TEE is around $0.1 \%$, in addition to the economic and personnel costs involved (10-13).

Consequently, there have been several attempts to define subsets of patients with $\mathrm{SAB}$ at low risk for IE, who could be safely managed omitting the performance of TEE. Recently, three clinical prediction scores have been published, PREDICT (14), VIRSTA (15) and POSITIVE (16). These scores can identify patients at low risk in which TEE could be deferred. To date, the scarce validation studies available have yielded controversial results (17-19). Moreover, all these studies have an important limitation: the low rate of echocardiography assessment, especially TEE in groups classified as low risk. It has been shown that the proportion of IE diagnosed increases with the number of patients with $\mathrm{SAB}$ who receive echocardiographic assessment $(20,21)$. Hence, it is likely that some patients had undiagnosed IE in these studies. This underdiagnosis could have an influence on mortality.

Despite this controversial results and limitations, some authors recommend forgoing echocardiographic evaluation (even TTE) in patients with no identifiable risk factor $(22,23)$. Furthermore, a recent scenario-based survey showed that approximately $15-20 \%$ of experts would recommend not performing TTE in patients with negative VIRSTA score (24).

Therefore, our primary objective was to evaluate the PREDICT, VIRSTA and POSITIVE scores, and to test their usefulness in safely ruling out IE without the need for TEE or TTE. The secondary objective was to determine, in patients without IE, the effect of withholding echocardiographic assessment on SAB mortality, in particular in those identified as low risk for IE, considering that missing an IE diagnosis could potentially cause an excess mortality.

\section{Materials and Methods}

We conducted a retrospective single-center study from January 2015 to December 2020. Our center is a third level university hospital with 613 beds in Madrid and a target population of 550,000 inhabitants.

Through a microbiology database, we identified all patients older than 18 years with a first (index) BC positive for SA during the study period. Patients with previous episodes of $\mathrm{SAB}$ were excluded. Index and follow-up BCs were obtained at discretion of the attending physician. BC were processed using the BD BACTEC FX system (Becton Dickinson, Sparks, Maryland, USA). When BC were positive, the strain was identified by MALDITOF (Bruker DaltonicTM). All systems were applied according to the manufacturer's instructions.

Data were collected retrospectively from the electronic medical record, including demographic, comorbidities, clinical, microbiological, echocardiographic and outcome data.

The study was approved by the hospital ethics committee. Since this was a retrospective, non-interventional study and only required collection of previously generated and anonymized data, informed consent was not required.

\subsection{Definitions}

IE was defined according to modified Duke criteria for definite IE (6). Bacteremia was considered persistent when follow-up BC were positive at least 48 hours after the extraction of the index BC despite appropriate antibiotic therapy, accordingly to the scores development studies definition (14-16). Complicated bacteremia was defined according to 
current guidelines (9). Primary bacteremia was defined as that in which the original source could not be determined. Sepsis and septic shock were defined according to current guidelines (25). Relapse was defined as the appearance of a new SAB at least 15 days after negative follow-up BC or 30 days after the extraction of the index BCs and initial clinical resolution in the absence of follow-up BCs.

\subsection{Predefined Ruling out IE thresholds}

IE was considered ruled out when the risk of IE was low enough for the patient not to benefit from echocardiographic assessment. In defining cutoff points for this low IE risk, we considered recent publications that estimate the risk of IE beyond which a patient does benefit from such assessment $(8,26)$. We also considered the usefulness of the negative likelihood ratio (NLR) to predict this risk, as previously reported (27). In accordance with the aforementioned studies, we predefined the following cut-off points: 1- If the risk of IE was less than $1 \%$ and the NLR was less than 0.05 , IE would be considered ruled out without the need for any echocardiographic assessment; 2- If the risk of IE was between $1-2 \%$ and the NLR was less than 0.10 , IE would be considered ruled out with the use of TTE without TEE. 3- If the risk of IE was between 2-5\% and the NLR was less than 0.20, it would be considered uncertain if IE may possibly be ruled out with a negative TTE without performing TEE. 4- If the risk of IE was greater than 5\% or the NLR was greater than 0.20 , TEE would be considered necessary to rule out IE. Additionally, we considered that if the prevalence of endocarditis in low-risk patients with negative TTE was higher than $1.1 \%$, these patients would benefit from TEE, in accordance with other authors (8).

\subsection{Primary and secondary objectives}

Our primary purpose was to assess the prevalence of IE in patients identified as low risk by means of PREDICT, VIRSTA and POSITIVE score, as well as determine the NLR of these scores. Our secondary objective was to evaluate the association between not performing an echocardiogram and 30-day mortality.

\subsection{Statistical analysis}

Quantitative variables are presented as median and interquartile range (IQR) and qualitative variables are presented as percentages and absolute values.

For the primary objective, the PREDICT, VIRSTA and POSITIVE scores were validated by calculating their sensitivity, specificity, likelihood ratios, predictive values, and their area under the receiving operating curve (AUC). The percentage of patients identified as low risk by these scores and who finally had IE is provided, including its $95 \%$ confidence interval (CI). Sensitivity analyses were performed including different populations according to whether different echocardiogram modalities were performed. A score was considered valid to rule out IE, with or without ETT, when the percentage of IE in patients identified as low risk and the score's NLR were below the mentioned cut-off points.

For the secondary objective, patients with IE diagnosed were excluded. In order to mitigate survivor bias, we excluded those patients who died within 48 hours of index BC extraction. Univariate analysis of 30-day mortality was performed using chi-square for qualitative variables (or Fisher exact test) and Mann-Whitney U for quantitative variables. Multivariate logistic regression models were developed, including echocardiography assessment (TTE, TEE or either) and those clinically relevant variables identified as statistically significant in the univariate analysis. Bilateral $p$-values of less than 0.05 were considered statistically significant. All statistical analyses were performed using SPSS version 25 statistical software (SPSS Inc, IBM, Chicago, Illinois, United States).

\section{Results}


During the study period, 404 patients with first episodes of SAB were identified. TTE was performed in $62.3 \%$ (250) and TEE in $32.2 \%$ (128). 50 patients (12.4\%) met modified Duke criteria for definite IE. Baseline characteristics, clinical, microbiological, imaging and outcome are summarized in Table 1.

Table 1. Factors associated with IE in patients with SAB.

\begin{tabular}{|c|c|c|c|c|c|c|}
\hline \multicolumn{2}{|c|}{ Variable } & Total $(n=404)$ & IE $(n=50)$ & Non-IE $(n=354)$ & $p$ & Missing \\
\hline \multicolumn{7}{|c|}{ Demographic and comorbidity } \\
\hline \multicolumn{2}{|c|}{ Age } & $69(56-79)$ & $68(58-77)$ & $69(55-80)$ & 0.927 & 0 \\
\hline \multicolumn{2}{|c|}{ Sex (female) } & $31.4 \%(127)$ & $26.0 \%(13)$ & $32.2 \%(1149$ & 0,420 & 0 \\
\hline \multicolumn{2}{|c|}{ Charlson index } & $2(1-5)$ & $2(1-4)$ & $2(1-5)$ & 0.635 & 3 \\
\hline \multicolumn{2}{|c|}{ Age-adjusted Charlson index } & $5(3-7)$ & $5(3-7)$ & $5(3-7)$ & 0.968 & 3 \\
\hline \multicolumn{2}{|c|}{ Arterial hypertension } & $55.9 \%(226)$ & $72.0 \%(36)$ & $53.7 \%(190)$ & 0.015 & 0 \\
\hline \multicolumn{2}{|c|}{ Diabetes mellitus } & $29.2 \%(118)$ & $34.0 \%(17)$ & $28.5 \%(101)$ & 0.506 & 0 \\
\hline \multicolumn{2}{|c|}{ Chronic heart failure } & $30.4 \%(123)$ & $48.0 \%(24)$ & $28.0 \%(99)$ & 0.005 & 0 \\
\hline \multicolumn{2}{|c|}{ Ischemic heart disease } & $18.1 \%(73)$ & $22.0 \%(11)$ & $17.5 \%(62)$ & 0.556 & 0 \\
\hline \multicolumn{2}{|c|}{ Natural cardiac valve disease } & $18.3 \%(74)$ & $29.7 \%(22)$ & $14.7 \%(52)$ & $<0.001$ & 0 \\
\hline \multicolumn{2}{|c|}{ Prosthetic heart valve disease } & $4.7 \%(19)$ & $14.0 \%(7)$ & $3.4 \%(12)$ & 0.005 & 0 \\
\hline \multicolumn{2}{|c|}{ CIED } & $6.2 \%(25)$ & $18.0 \%(9)$ & $4.5 \%(16)$ & 0.001 & 0 \\
\hline \multicolumn{2}{|c|}{ Chronic renal failure } & $22.8 \%(92)$ & $28.0 \%(14)$ & $22.0 \%(78)$ & 0.369 & 0 \\
\hline \multicolumn{2}{|c|}{ Hemodialysis } & $7.1 \%(29)$ & $8.0 \%(4)$ & $7.1 \%(25)$ & 0.794 & 0 \\
\hline \multicolumn{2}{|c|}{ Liver cirrhosis } & $3.2 \%(13)$ & $2.0 \%(1)$ & $3.4 \%(12)$ & 0.715 & 1 \\
\hline \multicolumn{2}{|c|}{ Solid organ malignancy } & $21.6 \%(87)$ & $12.0 \%(6)$ & $22.9 \%(81)$ & 0.098 & 1 \\
\hline \multirow{2}{*}{\multicolumn{2}{|c|}{ Parenteral drug user }} & $1.0 \%(4)$ & $2.0 \%(1)$ & $0.8 \%(3)$ & 0.413 & 2 \\
\hline & & \multicolumn{2}{|c|}{ Clinical presentation } & & & \\
\hline \multirow{4}{*}{ Acquisition } & Nosocomial & $51.5 \%(208)$ & $40.0 \%(20)$ & $53.1 \%(188)$ & Ref. & \\
\hline & $\begin{array}{l}\text { Healthcare } \\
\text { associated }\end{array}$ & $16.8 \%(68)$ & $14.0 \%(7)$ & $17.2 \%(61)$ & 0.870 & 0 \\
\hline & Community & $31.7 \%(128)$ & $46.0 \%(23)$ & $29.7 \%(105)$ & 0.026 & \\
\hline & Primary/unknown & $29.4 \%(116)$ & $49.0 \%(24)$ & $26.7 \%(92)$ & $<0.001$ & \\
\hline \multirow[t]{2}{*}{ Source of infection } & Catheter-related & $34.2 \%(135)$ & $28.0 \%(14)$ & $35.2 \%(121)$ & 0.571 & 10 \\
\hline & Other & $36.3 \%(143)$ & $24.0 \%(12)$ & $38.1 \%(131)$ & ref & \\
\hline \multicolumn{2}{|c|}{ Fever } & $89.8 \%(362)$ & $92.0 \%(46)$ & $89.5 \%(316)$ & 0.635 & 1 \\
\hline \multicolumn{2}{|c|}{ Sepsis/septic shock } & $28.3 \%(114)$ & $48.0 \%(24)$ & $25.5 \%(90)$ & 0.001 & 1 \\
\hline \multicolumn{2}{|c|}{ Fever defervescence within $72 \mathrm{~h}$} & $89.2 \%(330)$ & $79.2 \%(38)$ & $90.6 \%(292)$ & 0.050 & 34 \\
\hline Septic & emboli & $13.4 \%(54)$ & $46.0 \%(23)$ & $8.8 \%(31)$ & $<0.001$ & 0 \\
\hline Acute kid & ney injury & $40.5 \%(162)$ & $60.0 \%(30)$ & $37.7 \%(132)$ & 0.003 & 0 \\
\hline Acute carc & liac failure & $20.9 \%(84)$ & $54.0 \%(27)$ & $16.2 \%(57)$ & $<0.001$ & 2 \\
\hline Pitt's bacte & remia score & $0(0-3)$ & $1(0-3)$ & $0(0-2)$ & 0.004 & 2 \\
\hline $\mathrm{SO}$ & FA & $2(0-4)$ & $3(1-5)$ & $2(0-4)$ & 0.038 & 3 \\
\hline & & Microb & $\log y$ & & & \\
\hline Time to posi & tivity (hours) & $12(9-16)$ & $11(8-14)$ & $12(10-16)$ & 0.023 & 0 \\
\hline Persistent 1 & acteriemia & $31.8 \%(99)$ & $62.2 \%(28)$ & $26.7 \%(71)$ & $<0.001$ & 93 \\
\hline Meticilin-re & sistant SAB & $19.6 \%(79)$ & $14.0 \%(7)$ & $20.3 \%(72)$ & 0.345 & 0 \\
\hline & & Diagnosti & rork-up & & & \\
\hline $\mathrm{T}^{\mathrm{T}}$ & & $62.3 \%(250)$ & $80.0 \%(40)$ & $59.8 \%(210)$ & 0.007 & 3 \\
\hline $\mathrm{Tl}$ & & $32.2 \%(128)$ & $72.0 \%(36)$ & $26.4 \%(92)$ & $<0.001$ & 6 \\
\hline PET & $-\mathrm{CT}$ & $10.2 \%(41)$ & $26.0 \%(13)$ & $8.0 \%(28)$ & $<0.001$ & 3 \\
\hline & & Outc & & & & \\
\hline 30-day r & nortality & $15.4 \%(62)$ & $20.0 \%(10)$ & $14.8 \%(52)$ & 0.401 & 2 \\
\hline In-hospita & 1 mortality & $20.3 \%(82)$ & $28.0 \%(14)$ & $19.3 \%(68)$ & 0.187 & 0 \\
\hline
\end{tabular}




$\begin{array}{cccccc}\text { SAB relapse } & 4.5 \%(17) & 4.5 \%(2) & 4.5 \%(15) & 1.000 & 62\end{array}$

IE: infective endocarditis. Qualitative variables are presented as percentages (absolute number) and analyzed by means of Chi-square test (or Fisher exact test when necessary). Quantitative variables are presented as mediant (interquartile range) and analyze by means of Mann-Whitney's U. SAB: Staphylococcus aureus bacteriemia. CIED: Cardiac implantable electronic device. SOFA: Sepsis-related Organ Failure Assessment. TTE: transthoracic echocardiography. TEE: Transesophageal cardiography. PET-CT: Positron-Emission-Tomography Computed-Tomography.

\subsection{PREDICT score (day 5 model) evaluation}

Using the PREDICT score, $33.7 \%$ of patients (137/404) were identified as low risk. Of these, $3.6 \%$ had IE (Table 2), with a 95\% CI exceeding 5\% (0.1-6.9\%). The validation of the score is shown in table 3 and figure 1. PREDICT score had a NLR of 0.27 . According to the predefined cut-off points, employing this score would not be sufficient to safely rule out IE without TEE.

Table 2. IE and non-IE case distribution according to clinical prediction scores classification.

\begin{tabular}{ccccc}
\hline & & PREDICT (5-day model) & POSITIVE & VIRSTA \\
\hline \multirow{2}{*}{ High risk } & IE & $16.7 \%(45)$ & $23.6 \%(38)$ & $20.8 \%(46)$ \\
patients & Non IE & $83,3 \%(224)$ & $76,4 \%(123)$ & $79.2 \%(181)$ \\
& Total & $66.3 \%(269)$ & $39.7 \%(161)$ & $54.4 \%(221)$ \\
Low risk & IE & $3.6 \%(5)$ & $4.9 \%(12)$ & $2.2 \%(4)$ \\
patients & Non IE & $96.4 \%(132)$ & $95,1 \%(233)$ & $97.8 \%(181)$ \\
& Total & $33.7 \%(137)$ & $60.3 \%(245)$ & $45.6 \%(185)$ \\
\hline
\end{tabular}

IE: infective endocarditis.

Table 4 shows sensitivity analyses with the percentage of IE in patients with negative PREDICT score in different population according to echocardiogram evaluation. Of the low-risk patients with a negative TTE, 3.4\% (3/89) were eventually diagnosed with IE.

Table 3. Validation of different clinical prediction rules to identify IE among SAB patients.

\begin{tabular}{ccccccccc}
\hline & Cut-off & Sens. & Spec. & PPV & NPV & PLR & NLR & AUC \\
\hline $\begin{array}{c}\text { PREDICT (5-day } \\
\text { model) }\end{array}$ & $\mathbf{1}$ point & $90 \%$ & $37,1 \%$ & $16,7 \%$ & $96,4 \%$ & 1,43 & 0,27 & 0,70 \\
POSITIVE & $>$ 4 points & $76 \%$ & $65,5 \%$ & $23,6 \%$ & $95,1 \%$ & 2,17 & 0,37 & 0,78 \\
VIRSTA & $>$ 2 points & $92,0 \%$ & $50,8 \%$ & $20,8 \%$ & $97,8 \%$ & 1,84 & 0,16 & 0,85 \\
\hline
\end{tabular}

IE: infective endocarditis. SAB: Staphylococcus aureus bacteriemia. Sens: sensitivity. Spec: Specificity. PPV: Positive Predictive Value. NPV: Negative Predictive Value. PLR: Positive likelihoodratio. NLR: Negative likelihood-ratio. AUC: Area under de curve. 

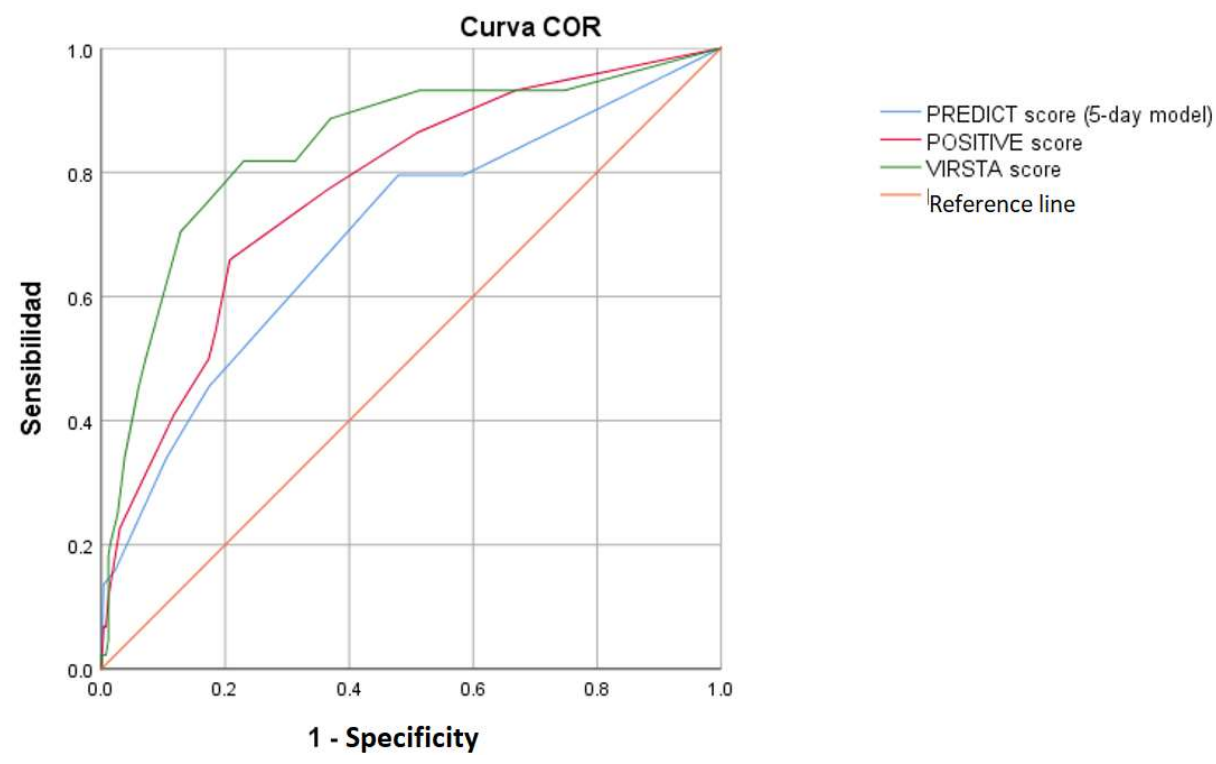

Figure 1. Receiving-Operator Curve of different clinical prediction rules. AUC for PREDICT score (5-day model) was 0.699 (IC 95\% 0.609-0.788). AUC for POSITIVE score was 0.771 (95\% CI 0.6960.846). AUC for VIRSTA score was 0.842 (0.771-0.912). AUC: Area Under de Curve.

\subsection{POSITIVE score evaluation}

Using the POSITIVE score, $60.3 \%$ of patients (245/404) were identified as low risk. Of these, $4.9 \%$ had IE (Table 2), with a 95\% CI exceeding 5\% (2.2-7.7\%). The validation of the score is shown in table 3 and figure S1. POSITIVE score had a NLR of 0.37. According to the predefined cut-off points, using the POSITIVE score would not be sufficient to rule out IE without a TEE.

Table 4 shows sensitivity analyses with the percentage of IE in patients with negative POSITIVE score in different population according to echocardiogram evaluation. Of the low-risk patients with a negative TTE, 2.8\% (4/141) were diagnosed with IE.

Table 4. IE rate among patients identified as low risk by different scores in different population according to echocardiographic evaluation and results.

\begin{tabular}{ccccccc}
\hline & \multicolumn{2}{c}{ PREDICT low risk } & \multicolumn{2}{c}{ POSITIVE low risk } & \multicolumn{2}{c}{ VIRSTA low risk } \\
\cline { 2 - 7 } & IE prevalence & CI 95\% & IE prevalence & CI 95\% & IE prevalence & CI 95\% \\
All patients $(\boldsymbol{n}=\mathbf{4 0 4 )}$ & $3.6 \%(5 / 137)$ & $0.1-6.9 \%$ & $4.9 \%(12 / 245)$ & $2.2 \%-7.7 \%$ & $2.2 \%(4 / 185)$ & $0.1-4.3 \%$ \\
Patients with TTE and/or & $4.8 \%(5 / 104)$ & $0.1-9.0 \%$ & $7.5 \%(12 / 160)$ & $3.4 \%-11.6 \%$ & $3.5 \%(4 / 116)$ & $0-6.8 \%$ \\
TEE $(\boldsymbol{n}=\mathbf{2 8 9 )}$ & $5.3 \%(2 / 39)$ & $0-12.4 \%$ & $12.0 \%(6 / 50)$ & $2.7 \%-21.3 \%$ & $5.7 \%(2 / 35)$ & $0-13.8 \%$ \\
$\begin{array}{c}\text { Patients with TEE }(\boldsymbol{n}=\mathbf{1 2 8}) \\
\text { Patients with negative TTE } \\
(\boldsymbol{n}=\mathbf{2 3 5})\end{array}$ & $3.4 \%(3 / 89)$ & $0-7.2 \%$ & $2.8 \%(4 / 141)$ & $0.1-5.6 \%$ & $0.9 \%(1 / 103)$ & $0-2.8 \%$ \\
\hline
\end{tabular}

IE: infective endocarditis. TEE: Transesophageal echocardiography. TTE: transthoracic echocardiography.

\subsection{VIRSTA score evaluation}

Using the VIRSTA score, $45.6 \%$ of patients (185/404) were identified as low risk. Of these, $2.2 \%$ had IE (Table 2), with a 95\% CI not exceeding 5\% (2.2-4.3\%). The validation of the score is shown in table 3 and figure S1. VIRSTA score had a NLR of 0.16. According to the predefined cut-off points, it would be uncertain if using the VIRSTA score in conjunction with a negative TTE would allow IE to be safely ruled out without a TEE. 
Table 4 shows sensitivity analyses with the percentage of IE in patients with negative VIRSTA score in different population according to echocardiogram evaluation. Of the low-risk patients with a negative TTE, $0.9 \%$ (1/103) were diagnosed with IE.

Supplementary Table S1 shows the patient-level data for SAB episodes identified as low risk by the VIRSTA score and finally diagnosed as IE. Only one patient had an identifiable risk factor (community-acquired bacteremia).

\subsection{Echocardiographic assessment and 30-day mortality}

After excluding patients diagnosed with IE and those who died within 48 hours after index BC extraction, performing an echocardiographic assessment was independently associated with lower 30-day mortality in a multivariate logistic regression model, including adjustment for VIRSTA score (OR 0.24 95\%CI 0.10-0.54). The model is shown in Table 5. Univariate analysis for the selection of variables included is shown in supplementary Table S2.

Table 5. Multivariate logistic regression model of mortality and echocardiographic evaluation.

\begin{tabular}{|c|c|c|c|c|}
\hline \multicolumn{2}{|c|}{ 30-day mortality } & OR & $95 \%$ CI & $p$ \\
\hline \multicolumn{2}{|c|}{ Age (each year) } & 1.04 & $1.01-1.07$ & 0.008 \\
\hline \multicolumn{2}{|c|}{ Charlson index (each point) } & 1.07 & $0.95-1.23$ & 0.329 \\
\hline \multicolumn{2}{|c|}{ Unknown source of infection } & 3.70 & $1.67-8.20$ & 0.001 \\
\hline \multicolumn{2}{|c|}{ SOFA (each point) } & 1.22 & $1.03-1.46$ & 0.026 \\
\hline \multicolumn{2}{|c|}{ Complicated bacteriemia } & 2.69 & $1.18-6.17$ & 0.019 \\
\hline \multicolumn{2}{|c|}{ Low risk VIRSTA score } & 0.44 & $0.19-0.99$ & 0.048 \\
\hline \multirow{3}{*}{$\begin{array}{c}\text { Echocardiographic } \\
\text { evaluation }\end{array}$} & TTE and/or TEE & 0.24 & $0.10-0.54$ & 0.001 \\
\hline & $T T E$ & 0.28 & $0.13-0.60$ & 0.001 \\
\hline & $T E E$ & 0.59 & $0.25-1.39$ & 0.232 \\
\hline
\end{tabular}

Only one echocardiographic variable at the time was included in the model. OR 95\% CI and $p$ values for variables other than echocardiographic evaluation are provided with the model including ETT and/or ETE. No significant different were observed within these variables when including the others echocardiographic evaluation. SOFA: Sepsis-related organ failure assessment. TTE: Transthoracic echocardiography. TEE: Transesophageal echocardiography. OR: Odds ratio. 95\% CI: 95\% confident interval.

30-day mortality in patients classified as low risk by VIRSTA score was lower if they had undergone TTE (5.1\% vs $15.7 \%, p=0.031)$.

\section{Discussion}

In our study, we evaluated whether the application of different scores for predicting IE risk in patients with SAB, with or without TTE, could safely rule out IE without TEE. Our main conclusion is that neither the PREDICT nor the POSITIVE scores would be sufficient to rule out IE without TEE, even with negative TTE. It was uncertain whether a negative VIRSTA score together with a negative TTE could allow to rule out IE without the need for TEE.

The PREDICT score was the first to be published (14). Its high negative predictive value was ratified in a validation study performed by the same group (28). However, other authors have found that this score does not identify low-risk patients with sufficient accuracy $(18,19)$. These results are in line with ours. It should be noted that PREDICT score does not include among its variables an important set of risk factors for IE (such as prosthetic heart valve), which could reduce its sensitivity (29). In summary, the application of the PREDICT score to avoid performance of TEE in SAB should not be recommended.

The POSITIVE score (16) is largely based on the shorter time to positivity of BC in the case of IE. In our study, as well as in other previous work (30), despite being a risk factor for IE, the association of time to positivity with IE was not as strong as that presented by 
the POSITIVE score authors. In line with our findings, a recent study (19) also failed to validate this score. In summary, this score does not identify patients at low risk with enough precision to avoid performing a TEE, so that it should not be recommended for this purpose.

The VIRSTA score (15) incorporates the largest number of variables as compared to the previous. Consequently, some studies have shown a higher sensitivity $(18,19)$. Hence, a recent survey showed that $15-20 \%$ of experts would recommend not performing TTE, nor TEE, evaluation in patients identified as low risk this score. Yet, in our data, the VIRSTA score was not sufficient to rule out IE without performing an echocardiogram evaluation (incidence of IE in low-risk patients $2.2 \%$, with NLR 0.16), and it was doubtful if a negative VIRSTA score plus a negative TTE would be able to rule out IE without performing a TEE. It should be noted that most of the patients eventually diagnosed with IE that had been identified as low risk by the VIRSTA score did not have any identifiable risk factors that would have raised IE suspicion. Ruling out IE in patients with negative VIRSTA and a negative TTE would allow to avoid TEE in approximately half of patients with SAB $(15,18,19)$. However, well-designed studies are needed to confirm the usefulness of this approach.

A potential restriction when applying these scores is that both their development and validation studies have been performed in cohorts without universal echocardiographic assessment, and only a small proportion of patients have undergone TEE. Several authors have demonstrated that carrying out an echocardiogram in a larger proportion of patients implies a higher rate of IE diagnoses $(20,21,31,32)$. It has even been proposed that performing an echocardiogram in these patients may be associated with lower mortality (33). In our study, we observed an increased 30-day mortality among patients with SAB who did not undergo an echocardiogram. We even found a higher mortality among patients identified as low risk for IE who had not undergo TTE. The fact that the association persists after adjusting for multiple variables, including comorbidity and severity of the bacteremia, supports the likelihood that this worse outcome was caused by the presence of undiagnosed occult IE, at least partially. Therefore, we decidedly believe that prospective studies validating these scores in a SAB cohort with systematic TTE and TEE is mandatory before assuming that they allow to avoid unnecessary echocardiography in low-risk patients.

Our study has some limitations. Firstly, as a single-center retrospective study, it presents the limitations of external validity that this entails. However, our population is comparable to that described in recent cohorts (34). Secondly, our predefined cut-off points are not prospectively validated. Nevertheless, both the IE risk percentages selected and the use of NLR have previously been proposed and accepted by several authors $(8,24,26,27,32)$. Thirdly, we cannot exclude that the higher 30 -day mortality found in patients with no echocardiogram is caused to uncontrolled confounding variables. However, the fact that this association persist after adjusting for several factors increases the likelihood that this worse outcome is ascribable, at least partially, to an undiagnosed IE. Finally, as mentioned, not all patients underwent echocardiography, and the percentage of patients lacking an echocardiogram was higher in low-risk patients. This absence of an echocardiogram, common in other studies as well, prevents the exclusion of occult IE in some of these patients, a fact that may limit the accuracy of the scores.

\section{Conclusions}

Neither the PREDICT nor the POSITIVE score were sufficient to exclude IE without concurrent TEE. In patients with negative VIRSTA score, it was uncertain whether IE could be discarded only with a negative TTE, without undergoing TEE. Moreover, we found an association between not performing echocardiographic assessment and an increased 30-day mortality, even in patients at low risk for IE, which could be related to the presence of occult IE. Hence, prospective well-designed studies with systematic 
performance of TTE and TEE are needed to verify whether these tests can be safely avoided in any subgroup of patients.

Supplementary Materials: The following supporting information can be downloaded at: www.mdpi.com/xxx/s1, Table S1: Individual characteristics of patients identified as low risk of IE by means of VIRSTA score but who finally were diagnosed of IE. Table S2: Univariate analysis of 30-day mortality in patients with no diagnosed with infective endocarditis and who survived the first 48 hours after the index blood culture extraction.

Author Contributions: Conceptualization, Jorge Calderón-Parra and Antonio Ramos-Martínez; Data curation, Jorge Calderón-Parra, Itziar Diego-Yagüe, Beatriz Santamarina-Alcantud, Alberto Mora-Vargas and Jose Manuel Vazquez-Comendador; Formal analysis, Jorge Calderón-Parra and Itziar Diego-Yagüe; Investigation, Jorge Calderón-Parra, Beatriz Santamarina-Alcantud, Alberto Mora-Vargas and Jose Manuel Vazquez-Comendador; Methodology, Jorge Calderón-Parra; Project administration, Jorge Calderón-Parra and Antonio Ramos-Martínez; Software, Jorge Calderón-Parra; Supervision, Ana Fernandez-Cruz, Elena Muñez-Rubio and Antonio Ramos-Martínez; Validation, Jorge Calderón-Parra, Ana Fernandez-Cruz and Antonio Ramos-Martínez; Writing - original draft, Jorge Calderón-Parra and Itziar Diego-Yagüe; Writing - review \& editing, Jorge CalderónParra, Itziar Diego-Yagüe, Ana Fernandez-Cruz, Elena Muñez-Rubio, Andrea Gutierrez-Villanueva, Isabel Sanchez-Romero and Antonio Ramos-Martínez

Funding: This research received no external funding.

Institutional Review Board Statement: The study was conducted in accordance with the Declaration of Helsinki and approved by the Institutional Review Board (or Ethics Committee) of Hospital Puerta de Hierro (protocol code PI 191/21).

Informed Consent Statement: Since this was a retrospective, non-interventional study and only required collection of previously generated and anonymized data, informed consent was not waived.

Data Availability Statement: The data presented in this study are available on request from the corresponding author.

Conflicts of Interest: The authors declare no conflict of interest.

\section{References}

1. Kuehl R, Morata L, Boeing C, Subirana I, Seifert H, Rieg S, et al. Defining persistent Staphylococcus aureus bacteraemia: secondary analysis of a prospective cohort study. Lancet Infect Dis. 2020 Dec;20(12):1409-17.

2. Le Moing V, Alla F, Doco-Lecompte T, Delahaye F, Piroth L, Chirouze C, et al. Staphylococcus aureus Bloodstream Infection and Endocarditis--A Prospective Cohort Study. PloS One. 2015;10(5):e0127385.

3. Hidalgo-Tenorio C, Gálvez J, Martínez-Marcos FJ, Plata-Ciezar A, De La Torre-Lima J, López-Cortés LE, et al. Clinical and prognostic differences between methicillin-resistant and methicillin-susceptible Staphylococcus aureus infective endocarditis. BMC Infect Dis. 2020 Feb 21;20(1):160.

4. Hsu R-B. Risk Factors for Nosocomial Infective Endocarditis in Patients with Methicillin-Resistant Staphylococcus Aureus Bacteremia. Infect Control Hosp Epidemiol [Internet]. 2005 Jul [cited 2021 Oct 13];26(7):654-7. Available from: https://www.cambridge.org/core/product/identifier/S0899823X00198605/type/journal_article

5. José Mensa, Alex Soriano, Pedro Llinares, José Barberán, Miguel Montejo, Miguel Salavert, et al. Guía de tratamiento antimicrobiano de la infección por Staphylococcus aureus. Rev Espanola Quimioter Publicacion Of Soc Espanola Quimioter. 2013;26 (Suppl. 1):1-84.

6. Habib G, Lancellotti P, Antunes MJ, Bongiorni MG, Casalta J-P, Del Zotti F, et al. 2015 ESC Guidelines for the management of infective endocarditis: The Task Force for the Management of Infective Endocarditis of the European Society of Cardiology (ESC)Endorsed by: European Association for Cardio-Thoracic Surgery (EACTS), the European Association of Nuclear Medicine (EANM). Eur Heart J [Internet]. 2015 Nov 21 [cited 2021 Sep 20];36(44):3075-128. Available from: https://academic.oup.com/eurheartj/article-lookup/doi/10.1093/eurheartj/ehv319

7. Gudiol F, Aguado JM, Almirante B, Bouza E, Cercenado E, Domínguez MÁ, et al. Diagnosis and treatment of bacteremia and endocarditis due to Staphylococcus aureus. A clinical guideline from the Spanish Society of Clinical Microbiology and Infectious Diseases (SEIMC). Enferm Infecc Microbiol Clin. 2015 Nov;33(9):625.e1-625.e23.

8. Heriot GS, Tong SYC, Cheng AC, Liew D. What risk of endocarditis is low enough to justify the omission of transoesophageal echocardiography in Staphylococcus aureus bacteraemia? A narrative review. Clin Microbiol Infect Off Publ Eur Soc Clin Microbiol Infect Dis. 2018 Dec;24(12):1251-6. 
9. Liu C, Bayer A, Cosgrove SE, Daum RS, Fridkin SK, Gorwitz RJ, et al. Clinical Practice Guidelines by the Infectious Diseases Society of America for the Treatment of Methicillin-Resistant Staphylococcus aureus Infections in Adults and Children. Clin Infect Dis [Internet]. 2011 Feb 1 [cited 2021 Oct 13];52(3):e18-55. Available from: https://academic.oup.com/cid/article/52/3/e18/306145

10. Hilberath JN, Oakes DA, Shernan SK, Bulwer BE, D'Ambra MN, Eltzschig HK. Safety of transesophageal echocardiography. J Am Soc Echocardiogr Off Publ Am Soc Echocardiogr. 2010 Nov;23(11):1115-27; quiz 1220-1.

11. Min JK, Spencer KT, Furlong KT, DeCara JM, Sugeng L, Ward RP, et al. Clinical features of complications from transesophageal echocardiography: a single-center case series of 10,000 consecutive examinations. J Am Soc Echocardiogr Off Publ Am Soc Echocardiogr. 2005 Sep;18(9):925-9.

12. Liu E, Guha A, Dunleavy M, Obarski T. Safety of Transesophageal Echocardiography in Patients with Esophageal Varices. J Am Soc Echocardiogr Off Publ Am Soc Echocardiogr. 2019 May;32(5):676-7.

13. Kitkungvan D, Kalluru D, Lunagariya A, Sanchez L, Yusuf SW, Hassan S, et al. Safety of Transesophageal Echocardiography in Patients with Thrombocytopenia. J Am Soc Echocardiogr Off Publ Am Soc Echocardiogr. 2019 Aug;32(8):1010-5.

14. Palraj BR, Baddour LM, Hess EP, Steckelberg JM, Wilson WR, Lahr BD, et al. Predicting Risk of Endocarditis Using a Clinical Tool (PREDICT): Scoring System to Guide Use of Echocardiography in the Management of Staphylococcus aureus Bacteremia. Clin Infect Dis [Internet]. 2015 Jul 1 [cited 2021 Oct 13];61(1):18-28. Available from: https://academic.oup.com/cid/articlelookup/doi/10.1093/cid/civ235

15. Tubiana S, Duval X, Alla F, Selton-Suty C, Tattevin P, Delahaye F, et al. The VIRSTA score, a prediction score to estimate risk of infective endocarditis and determine priority for echocardiography in patients with Staphylococcus aureus bacteremia. J Infect [Internet]. 2016 May [cited 2021 Oct 13];72(5):544-53. Available from: https://linkinghub.elsevier.com/retrieve/pii/S0163445316000517

16. Kahn F, Resman F, Bergmark S, Filiptsev P, Nilson B, Gilje P, et al. Time to blood culture positivity in Staphylococcus aureus bacteraemia to determine risk of infective endocarditis. Clin Microbiol Infect [Internet]. 2021 Sep [cited 2021 Oct 13];27(9):1345.e7-1345.e12. Available from: https://linkinghub.elsevier.com/retrieve/pii/S1198743X2030700X

17. Bai AD, Agarwal A, Steinberg M, Showler A, Burry L, Tomlinson GA, et al. Clinical predictors and clinical prediction rules to estimate initial patient risk for infective endocarditis in Staphylococcus aureus bacteraemia: a systematic review and metaanalysis. Clin Microbiol Infect [Internet]. 2017 Dec [cited 2021 Oct 13];23(12):900-6. Available from: https://linkinghub.elsevier.com/retrieve/pii/S1198743X17302367

18. Peinado-Acevedo JS, Hurtado-Guerra JJ, Hincapié-Osorno C, Mesa-Abad J, Uribe-Delgado JR, Giraldo-Ramírez S, et al. Validation of VIRSTA and PREDICT scores to determine the priority of echocardiography in patients with Staphylococcus aureus bacteremia. Clin Infect Dis Off Publ Infect Dis Soc Am. 2021 Feb 4;ciaa1844.

19. Van der Vaart TW, Prins JM, Soetekouw R, van Twillert G, Veenstra J, Herpers BL, et al. Prediction rules for ruling out endocarditis in patients with Staphylococcus aureus bacteremia. Clin Infect Dis Off Publ Infect Dis Soc Am. 2021 Jul 16;ciab632.

20. Bolhuis K, Bakker LJ, Keijer JT, de Vries PJ. Implementing a hospital-wide protocol for Staphylococcus aureus bacteremia. Eur J Clin Microbiol Infect Dis [Internet]. 2018 Aug [cited 2021 Oct 13];37(8):1553-62. Available from: http://link.springer.com/10.1007/s10096-018-3284-9

21. Holden E, Bashir A, Das I, Morton H, Steadman CD, Nightingale P, et al. Staphylococcus aureus bacteraemia in a UK tertiary referral centre: a "transoesophageal echocardiogram for all” policy. J Antimicrob Chemother [Internet]. 2014 Jul 1 [cited 2021 Oct 13];69(7):1960-5. Available from: https://academic.oup.com/jac/article-lookup/doi/10.1093/jac/dku082

22. Joseph JP, Meddows TR, Webster DP, Newton JD, Myerson SG, Prendergast B, et al. Prioritizing echocardiography in Staphylococcus aureus bacteraemia. J Antimicrob Chemother. 2013 Feb;68(2):444-9.

23. Mun SJ, Kim S-H, Huh K, Cho SY, Kang C-I, Chung DR, et al. Role of echocardiography in uncomplicated Staphylococcus aureus catheter-related bloodstream infections. Medicine (Baltimore). 2021 May 7;100(18):e25679.

24. Heriot GS, Tong SYC, Cheng AC, Liew D. A Scenario-Based Survey of Expert Echocardiography Recommendations for Patients With Staphylococcus aureus Bacteremia at Varying Risk for Endocarditis. JAMA Netw Open. 2020 Apr 1;3(4):e202401.

25. Shankar-Hari M, Phillips GS, Levy ML, Seymour CW, Liu VX, Deutschman CS, et al. Developing a New Definition and Assessing New Clinical Criteria for Septic Shock: For the Third International Consensus Definitions for Sepsis and Septic Shock (Sepsis-3). JAMA. 2016 Feb 23;315(8):775-87.

26. Heriot GS, Tong SYC, Cheng AC, Liew D. Benefit of Echocardiography in Patients With Staphylococcus aureus Bacteremia at Low Risk of Endocarditis. Open Forum Infect Dis [Internet]. 2018 Dec 1 [cited 2021 Oct 13];5(12):ofy303. Available from: https://academic.oup.com/ofid/article/doi/10.1093/ofid/ofy303/5238173

27. Bai AD, Showler A, Burry L, Steinberg M, Tomlinson GA, Bell CM, et al. Clinical prediction rules in Staphylococcus aureus bacteremia demonstrate the usefulness of reporting likelihood ratios in infectious diseases. Eur J Clin Microbiol Infect Dis [Internet]. 2016 Sep [cited 2021 Oct 13];35(9):1393-8. Available from: http://link.springer.com/10.1007/s10096-016-2711-z

28. Abu Saleh O, Fida M, Asbury K, Narichania A, Sotello D, Bosch W, et al. Prospective Validation of PREDICT and Its Impact on the Transesophageal Echocardiography Use in Management of Staphylococcus aureus Bacteremia. Clin Infect Dis [Internet]. 2021 Oct 5 [cited 2021 Oct 13];73(7):e1745-53. Available from: https://academic.oup.com/cid/article/73/7/e1745/5860918 
29. Heriot GS, Cronin K, Tong SYC, Cheng AC, Liew D. Criteria for Identifying Patients With Staphylococcus aureus Bacteremia Who Are at Low Risk of Endocarditis: A Systematic Review. Open Forum Infect Dis [Internet]. 2017 Oct 1 [cited 2021 Oct 13];4(4):ofx261. Available from: https://academic.oup.com/ofid/article/doi/10.1093/ofid/ofx261/4657117

30. Siméon S, Le Moing V, Tubiana S, Duval X, Fournier D, Lavigne J-P, et al. Time to blood culture positivity: An independent predictor of infective endocarditis and mortality in patients with Staphylococcus aureus bacteraemia. Clin Microbiol Infect Off Publ Eur Soc Clin Microbiol Infect Dis. 2019 Apr;25(4):481-8.

31. Vos FJ, Bleeker-Rovers CP, Sturm PD, Krabbe PFM, Kullberg BJ. Endocarditis: effects of routine echocardiography during Gram-positive bacteraemia. Augus T. 2011;(7):6.

32. Lau L, Wiens EJ, Karlowsky JA, Keynan Y, Jassal DS. Clinical utility of echocardiography for the diagnosis of native valve infective endocarditis in Staphylococcus aureus bacteremia. Echocardiography [Internet]. 2019 Oct [cited 2021 Oct 13];36(10):1852-8. Available from: https://onlinelibrary.wiley.com/doi/10.1111/echo.14480

33. Urja P, Walters RW, Vivekanandan R, Kumar M, Abdulghani S, Hari Belbase R, et al. Trends in the use of echocardiography in patients with Staphylococcus aureus bacteremia: an analysis using the Nationwide Inpatient Sample data. Echocardiography [Internet]. 2019 Sep [cited 2021 Oct 13];36(9):1625-32. Available from: https://onlinelibrary.wiley.com/doi/10.1111/echo.14473

34. Lam JC, Gregson DB, Somayaji R, Robinson S, Conly JM, Welikovitch L, et al. Forgoing transesophageal echocardiogram in selected patients with complicated Staphylococcus aureus bacteremia. Eur J Clin Microbiol Infect Dis [Internet]. 2021 Mar [cited 2021 Oct 13];40(3):623-31. Available from: http://link.springer.com/10.1007/s10096-020-04097-y 